\title{
Pulmonary embolization with tunneled hemodialysis catheter-associated blood stream infection: the perils of systemic anticoagulation
}

\author{
Kamel A. Gharaibeh, Éva Csongrádi, ${ }^{2}$ Michael Shoemaker-Moyle, ${ }^{1}$ Anna Lerant, ${ }^{3}$ \\ Mihály Tapolyai, ${ }^{4}$ Tibor Fülöp ${ }^{1}$ \\ 'Department of Internal Medicine, University of Mississippi Health Care and University of \\ Mississippi Medical Center, Jackson, MS, USA; ${ }^{2}$ Department I of Medicine, Medical and Health \\ Science Center, University of Debrecen, Hungary; ${ }^{3}$ Department of Anesthesiology and Medical \\ Advanced Skill and Simulation Education Center, University of Mississippi Medical Center, \\ Jackson, MS, USA; ${ }^{4}$ Fresenius Medical Care, Semmelweis University, Budapest, Hungary
}

\begin{abstract}
A 35-year old African-American male with end-stage renal disease on hemodialysis through a tunneled dialysis catheter (TDC) presented with fever, diffuse aches and generalized distress. Blood cultures (BC) were obtained and empirical broad-spectrum antibi-
\end{abstract}

Correspondence: Kamel A. Gharaibeh, Department of Medicine, University of Mississippi Medical Center, 2500 North State Street, Jackson, Mississippi 39216-4505, USA. Tel. +1.601.984.5600 - Fax +1.601.984.5765 E-mail: kgharaibeh@umc.edu

Key words: bacteremia, cerebral hemorrhage, end-stage renal disease, hemodialysis catheter, pulmonary embolism.

Received for publication: 31 December 2011. Revision received: 20 June 2012

Accepted for publication: 25 June 2012.

Conflict of interests: the authors report no conflict of interests.

Contributions: KAG, data collection, chart review, initial draft of paper and correspondence; EC, manuscript revision, clinical relevance and literature review; $\mathrm{AL}$, manuscript revision and data presentation; MS-M critical manuscript revision and clinical relevance of observation; MT, expert revision and review; TF, case identification, manuscript development, coordinating manuscript revisions and senior author.

This work is licensed under a Creative Commons Attribution NonCommercial 3.0 License (CC BYNC 3.0).

(C) Copyright K.A. Gharaibeh et al., 2012

Licensee PAGEPress, Italy

Nephrology Reviews 2012; 4:e17

doi:10.4081/nr.2012.e17 otic therapy started. After urgent renal dialysis, TDC was pulled at the bedside. Chest computed tomography (CT) diagnosed pulmonary embolism and systemic i.v. heparin was initiated. BC grew Gram positive cocci (methicillinsensitive $S$. aureus) and cardiac echocardiogram confirmed acute bacterial endocarditis. Due to declined mental status, CT imaging was obtained revealing massive intracranial hemorrhage leading to the patient's death. Further chest CT review revealed only hemodynamically non-significant pulmonary emboli in some segmental and subsegmental arteries. Pulmonary embolization may be an expected occurrence with removal of infected TDC; systemic anticoagulation may not be warranted in such cases and may lead to catastrophic intracranial hemorrhage. This case draws our attention to the perils of rigid adherence to protocol and the failure of considering the needs of special patient cohorts and individualized care.

\section{Introduction}

A 35 year-old functional African-American male with end-stage renal disease (ESRD) on hemodialysis was admitted with fever, shortness of breath, and diffuse joint and body aches of three days' duration. His past medical history included high blood pressure and lupus nephritis that had led to ESRD five years earlier. He had received a deceased-donor renal transplant two and a half years ago with three subsequent episodes of acute cellular and humoral rejections. Ultimately, his renal allograft failed eight months prior to admission and he had to resume regular renal hemodialysis utilizing a right internal jugular tunneled dialysis catheter (TDC). Immunomodulating therapy had been discontinued for six months prior to presentation. A right forearm arterio-venous fistula (AVF) had been placed four months ago and was awaiting further procedures to assist maturation.

\section{Case Report}

On admission, the patient's vital signs included a temperature of $39.3^{\circ} \mathrm{C}$, blood pressure $152 / 58 \mathrm{mmHg}$ with a heart rate of 133 beats/min and respiratory rate $18 / \mathrm{min}$. Oxygen saturation was $97 \%$ on room air. General physical examination revealed mild confusion (oriented to person and place only) and he was unwilling to lay flat. Cardiovascular examination showed a displaced left apex with regular tachycardia but no rubs or gallops. His lung fields were clear on auscultation and he had no edema. A right internal jugular TDC had no external signs of infection. Laboratory tests showed anemia with hemoglobin of $8.9 \mathrm{gm} / \mathrm{dL}$ and an elevated WBC count $\left(15.6 \times 10^{9} / \mathrm{L}\right)$ with neutrophilia (92\% segmented neutrophils). Arterial blood gases showed hypoxia with p02 $83 \mathrm{mmHg}$, pCO2 $41 \mathrm{mmHg}$ and PH 7.39 while receiving $\mathrm{O}_{2}$ supply at $10 \mathrm{~L} / \mathrm{min}$ via a face mask. C-reactive protein (CRP) was markedly elevated $(28.1 \mathrm{mg} / \mathrm{dL}$; reference value $<0.49)$ along with elevated procalcitonine (PCT) $(>40$ $\mathrm{ng} / \mathrm{mL}$; reference value $<0.05)$. In the emergency room, blood cultures were obtained and broad-spectrum antibiotics (vancomycin, tobramycin, and levofloxacin) started. The patient received urgent hemodialysis with subsequent uncomplicated bedside removal of TDC. Initial blood cultures grew Gram positive cocci (finalized as methicillin-sensitive $S$. aureus). Overnight, the patient's condition deteriorated with decreasing blood pressure, increasing heart rate, and worsening hypoxia and respiratory distress. He was transferred to the intensive care unit (ICU) with subsequent intubation and mechanical ventilation. Chest X-ray showed bilateral basilar densities. Intravenous heparin was initiated for suspected pulmonary embolization, confirmed on contrast-enhanced computed tomography (CT) of the chest. Transthoracic and transeosophagial cardiac echocardiogram revealed aortic and mitral valve acute bacterial endocarditis with 
valvular insufficiency. Right ventricular size and function were normal with an estimated pulmonary pressure of $31 \mathrm{mmHg}$. Blood cultures remained positive despite continued antibiotics. The treatment course was complicated by worsening anemia, thrombocytopenia and the need to restart renal replacement therapy with continuous veno-venous hemofiltration and regional citrate anticoagulation. A cardiothoracic surgeon was consulted but it was felt that the patient was not a candidate for surgery due to the severity of the pathology. On ICU Day 7, his central nervous system status suddenly deteriorated; neuroimaging (MRI) revealed massive intracranial hemorrhage (Figures 1 and 2) with impending transtentorial herniation. The patient's family was consulted and it was decided that he would receive comfort care only; the patient died peacefully on hospital Day 12 . A further review of the pulmonary angiogram confirmed that

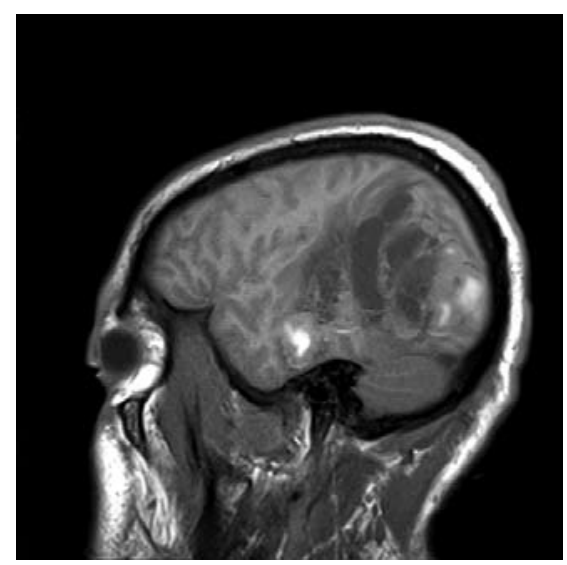

Figure 1. Brain magnetic resonance imaging with bleed, saggittal view reconstruction.

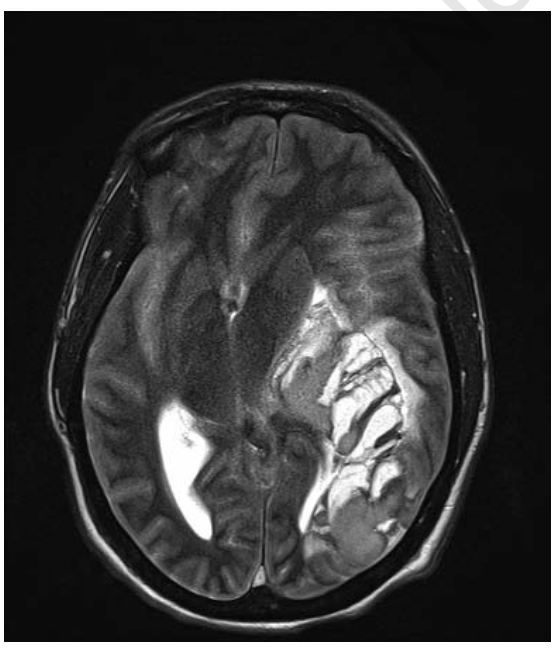

Figure 2. Brain magnetic resonance imaging with midline shift and bleed. the observed segmental and subsegmental arterial pulmonary emboli were unlikely to be hemodynamically significant (Figures $3 \mathrm{~A}$ and 3B). He also had diffuse central cavitations in the lung bases consistent with an infectious/ inflammatory process.

\section{Discussion}

Delay in removal of the infected hardware (TDC) may increase the risk of infective endocarditis in general; but this was not the case here. Upon arrival, the initial differential diagnosis for this patient included acute flare of systemic lupus, creating confusion. Markedly elevated serological markers of inflammation (CRP, PCT) promoted early differentiation of an SLE form acute bacterial infection before blood cultures confirmed positivity. Bedside removal of infected TDC was accomplished early on and, in our experience, can be accomplished successfully with minimal complication rates even by nephrology trainees. ${ }^{1}$ In hindsight, primary peritoneal dialysis ( $P D$ first) would have been the best option to offer the patient renal dialysis while awaiting AVF placement and maturation. Some of these patients can be continued on low-dose immunomodulating therapy to preserve residual renal function that may help to facilitate bridging peritoneal dialysis. Pulmonary embolization in ESRD is a rare event and should prompt a diligent search for the underlying cause. ${ }^{2}$ In fact, in patients with infected TDC, one would expect some clot formation on the catheter tip which, upon removal of the catheter, would lodge into the pulmonary circu- lation. ${ }^{3,4}$ Embolization from subclavian or internal jugular vein are, however, unlikely to be of sufficient mass to compromise pulmonary perfusion to a lethal degree. Similarly, pulmonary thromboembolism is, in fact, a routine occurrence after thrombolysis of clotted $\mathrm{AV}$ grafts in dialysis patients and does not on its own demand routine anticoagulation. ${ }^{5}$ It is unlikely that hypoxia in this patient represented the pulmonary embolization but rather a worsening of the septic-inflammatory status with ongoing bacteremia and effective antibiotic therapy. Thrombosis in the femoral vein would, however, be expected to be different due to the femoral vein's large size and large clot load. ${ }^{6}$ While some argument for the safety of anticoagulation with endocarditis has been made in the general population, ${ }^{7}$ there are no such data for ESRD patients. The combination of systemic anticoagulation with heparin and acute bacterial endocarditis was, indeed, the worst possible confluence of events for this patient and likely contributed to the catastrophic intracranial bleed. Hemorrhagic transformation of septic emboli and the development of pyogenic arteriitis likely represented the mechanism for this observed catastrophic outcome. ${ }^{8}$ Other mechanisms of catheter related embolization also include a catheter-associated fibrous sheath or even a right atrial thrombus. ${ }^{9}$ The literature identifies no treatment choice to be superior over the other regarding anticoagulation, thus uncertainties and controversy over treatment remain. While clinical protocols can help reduce medical errors, they should be carefully supervised with a critical analysis of the needs of each individual patient.
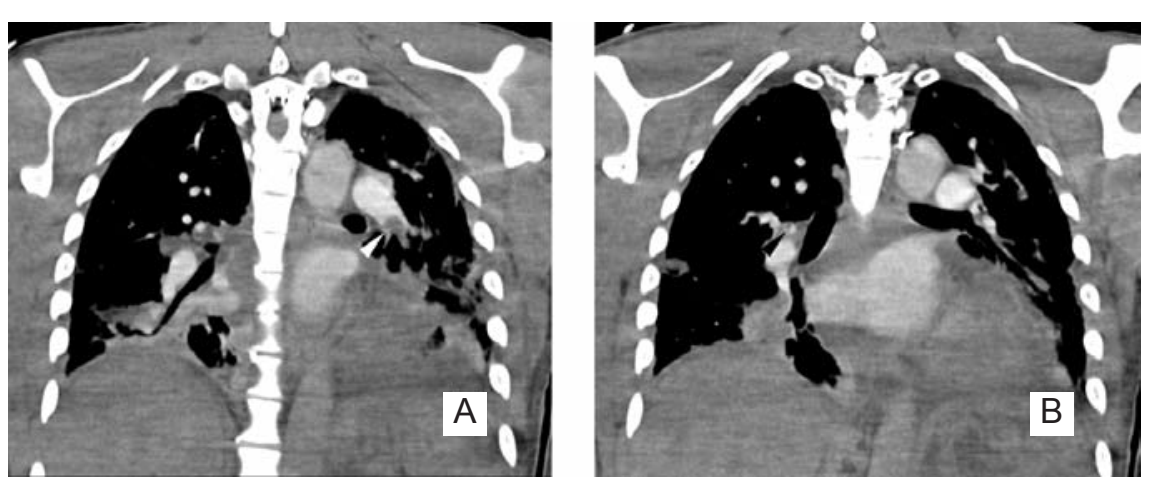

Figures 3. A,B) computed tomography angiogram of the chest with minor pulmonary embolism (arrowheads). 


\section{References}

1. Beemidi V, Qureshi NA, Dreisbach AW, et al. The safety and efficacy of bedside tunneled dialysis catheters removal by physician-in-training in an academic teaching setting. J Am Soc Nephrol 2011;22:568 A.

2. Mossey RT, Kasabian AA, Wilkes BM. Pulmonary embolism Low incidence in chronic renal failure. Arch Int Med 1982; 142:1646.

3. Jones MA, Lee DY, Segall JA, et al. Characterizing resolution of catheter associated upper extremity deep venous thrombosis. J Vasc Surg 2010;51:108-13.

4. Kooij JD, van der Zant FM, van Beek EJ, Reekers JA. Pulmonary embolism in deep venous thrombosis of the upper extremity: more often in catheter - related thrombosis. Netherlands J Med 1997;50:238.

5. Calderon K, Jhaveri KD, Mossey R. Pulmonary embolism following thrombolysis of dialysis access: is anticoagulation really necessary? Seminars Dial 2010;23: 522-5.

6. Hsieh HS, Liao HT, Wei CJ, Tarng DC. Indwelled femoral vein non - cuffed, double - lumen hemodialysis catheter complicated by pulmonary thromboembolism. Clin Nephrol 2004;62:162.
7. Rasmussen RV, Snygg-Martin U, Olaison L, et al. Major cerebral events in Staphylococcus aureus infective endocarditis: is anticoagulant therapy safe? Cardiology 2009;114:284-91.

8. Masuda J, Yutani C, Waki R, et al. Histopathological analysis of the mechanisms of intracranial hemorrhage complicating infective endocarditis. Stroke 1992; 23:843.

9. Stavroulopoulos A, Aresti V, Zounis C. Right atrial thrombi complicating haemodialysis catheters. A meta-analysis of reported cases and a proposal of a management algorithm Nephrol Dial Transplant 2012;27:2936-44. 